\title{
Método q-GC: uma generalização do método dos gradientes conjugados
}

\author{
Érica J. C. Gouvêa, Marluce Scarabello, \\ Programa de Doutorado em Computação Aplicada, CAP, INPE, \\ 12227-010, São José dos Campos, SP \\ E-mail: ericagouvea@gmail.com, marluce.scarabello@inpe.br,
}

\begin{abstract}
Aline C. Soterroni, Fernando Manuel Ramos, Laboratório Associado de Computação e Matemática Aplicada, LAC, INPE, 12227-010, São José dos Campos, SP

E-mail: alinecsoterroni@gmail.com, fernando.ramos@inpe.br,
\end{abstract}

\section{Roberto L. Galski}

Centro de Rastreio e Controle de Satélites, CRC, INPE

12210-080, São José dos Campos, SP

E-mail: galski@ccs.inpe.br .

\begin{abstract}
Resumo: Recentemente, baseado na derivada de Jackson, foi proposta uma generalização do método da máxima descida, denominada método do q-gradiente (q-G), para problemas de otimização global contínua. Dentro desse contexto, este trabalho apresenta uma generalização do método dos gradientes conjugados $(q-G C)$ com base no conceito do vetor q-gradiente. Para avaliar o desempenho do método q-GC foram considerados os resultados obtidos pelo método q-G e por três Algoritmos Genéticos (AGs) para um conjunto de seis funções teste de 20 variáveis e mesmo critério de parada. No geral, os resultados mostram que o q-GC é um método promissor para solução de problemas de otimização multimodais.
\end{abstract}

Palavras-chave: q-gradiente, q-derivada, método q-G, método q-GC

\section{Introdução}

Recentemente, uma generalização do método da máxima descida, denominada método q-G, foi desenvolvida por [10] para problemas de otimização global contínua. Esse método utiliza como direção de busca, a direção contrária à direção do vetor $q$-gradiente, definido a partir da derivada clássica com o auxílio do parâmetro $q$. Dentro desse contexto, esse trabalho apresenta uma generalização do método dos gradientes conjugados, denominada método dos $q$-Gradientes Conjugados ( $q$-GC), onde a primeira direção de busca é a direção contrária à direção do vetor $q$-gradiente e as outras direções são combinações lineares da direção contrária à direção do vetor $q$-gradiente do ponto atual com as direções anteriores. Tanto no método $q$-G quanto no método $q$-GC, $q$ é um parâmetro chave e, à medida que $q$ tende a 1 , as $q$-versões retomam suas respectivas versões clássicas.

Para testar essa nova abordagem, o método q-GC foi aplicado em seis funções teste (três unimodais e três multimodais) comumente utilizadas na área de otimização contínua. Os resultados são comparados com os obtidos pelo método $q-\mathrm{G}$, além dos Algoritmos Genéticos (AGs) G3-PCX (veja [2]), SPC-vSBX e SPC-PNX (veja [1]). Embora preliminares, os resultados mostram a capacidade do método de escapar de mínimos locais em problemas multimodais. Já para 
os problemas unimodais, o q-GC permite encontrar o mínimo da função em menos iterações ou com uma precisão melhor que o método $q-\mathrm{G}$.

\section{O método $q-\mathrm{GC}$}

A derivada de Jackson, ou $q$-derivada, de uma função $f(x)$ de uma única variável é dada por (veja [6])

$$
D_{q} f(x)=\frac{f(q x)-f(x)}{q x-x},
$$

onde $q$ é um número real diferente de 1 e $x$ diferente de 0 . No limite, quando $q \rightarrow 1$ (ou $x \rightarrow 0$ ), a $q$-derivada retorna à derivada clássica. Para funções diferenciáveis de $n$ variáveis, $f(\mathbf{x})$, o $q$-gradiente é o vetor das $n q$-derivadas parciais de primeira ordem de $f$ dado por (veja [10])

$$
D_{q_{i}, x_{i}} f(\mathbf{x})=\left\{\begin{array}{ll}
\frac{f\left(x_{1}, \ldots, q_{i} x_{i}, \ldots, x_{n}\right)-f\left(x_{1}, \ldots, x_{i}, \ldots, x_{n}\right)}{q_{i} x_{i}-x_{i}}, & x_{i} \neq 0 \text { e } q_{i} \neq 1 \\
\frac{\partial f(\mathbf{x})}{\partial x_{i}}, & x_{i}=0 \text { ou } q_{i}=1
\end{array},\right.
$$

onde o parâmetro $q$ é um vetor $\mathbf{q}=\left(q_{1}, \ldots, q_{i}, \ldots, q_{n}\right)$ com $q_{i} \neq 1, \forall i$. Note que, se $x_{i}=0$ ou $q_{i}=1$, a $q$-derivada parcial de primeira ordem retorna à derivada parcial clássica. A equação anterior define o vetor $q$-gradiente de $f$ como [10]

$$
\nabla_{\mathbf{q}} f(\mathbf{x})^{T}=\left[D_{q_{1}, x_{1}} f(\mathbf{x}) \ldots D_{q_{i}, x_{i}} f(\mathbf{x}) \ldots D_{q_{n}, x_{n}} f(\mathbf{x})\right]
$$

em que no limite, $q_{i} \rightarrow 1(\forall i=1, \ldots, n)$, o vetor $q$-gradiente retorna ao vetor gradiente clássico.

Em geral, os métodos de otimização utilizam o procedimento iterativo $\mathbf{x}^{k+1}=\mathbf{x}^{k}+\alpha^{k} \mathbf{d}^{k}$, em que $\mathbf{d}^{k}$ é a direção de busca e $\alpha^{k}$ é o tamanho do passo dado nesta direção na iteração $k$. Os métodos de otimização baseados em gradiente diferem entre si na forma em que a direção e o tamanho do passo são calculados. O método da máxima descida, por exemplo, utiliza $\mathbf{d}^{k}=-\nabla f\left(\mathbf{x}^{k}\right)$ como direção de busca e o tamanho do passo $\alpha^{k}$ é, em geral, determinado por uma técnica de busca linear que minimiza a função objetivo ao longo de $\mathbf{d}^{k}$. No método $q-\mathrm{G}$, a direção de busca é a direção contrária à direção do vetor q-gradiente da função objetivo no ponto $x^{k},-\nabla_{\mathbf{q}} f\left(\mathbf{x}^{k}\right)$, como definida na Equação (3). De forma similar, o método $q$-GC é uma generalização do método dos gradientes conjugados de Fletcher e Reeves [4] no qual a primeira direção de busca é a direção contrária à direção do vetor q-gradiente e as outras direções são combinações lineares da direção contrária à direção do vetor $q$-gradiente do ponto atual com as direções anteriores.

O parâmetro $q$ utilizado no cálculo da $q$-derivada pode ser qualquer número real diferente de 1. Assim como em [10], os valores de $q_{i}^{k} x_{i}^{k}, i=1, \ldots, n$, foram sorteados segundo uma distribuição gaussiana com média no ponto $x_{i}^{k}$ e desvio padrão $\sigma^{k}$. O desvio padrão é inicialmente diferente de zero e tende a zero ao longo do procedimento iterativo por meio da expressão $\sigma^{k+1}=\beta \sigma^{k}$ em que $\beta \in[0,1]$ é o fator de redução.

Geralmente, os métodos baseados em gradiente realizam busca linear a cada iteração para determinar o comprimento do passo a ser dado em uma direção que é de descida (veja [3]). Uma vez que a direção de busca do método $q$-GC pode ser tanto de descida quanto de subida, dependendo do valor do parâmetro q, o cálculo para o comprimento do passo na iteração $k$ é dado por $\alpha^{k+1}=\beta \alpha^{k}$. Por simplicidade, $\beta$ é o mesmo fator de redução usado no cálculo do $\sigma^{k}$ (veja [10]).

As principais etapas do algoritmo do método $q$-GC são descritas a seguir. 


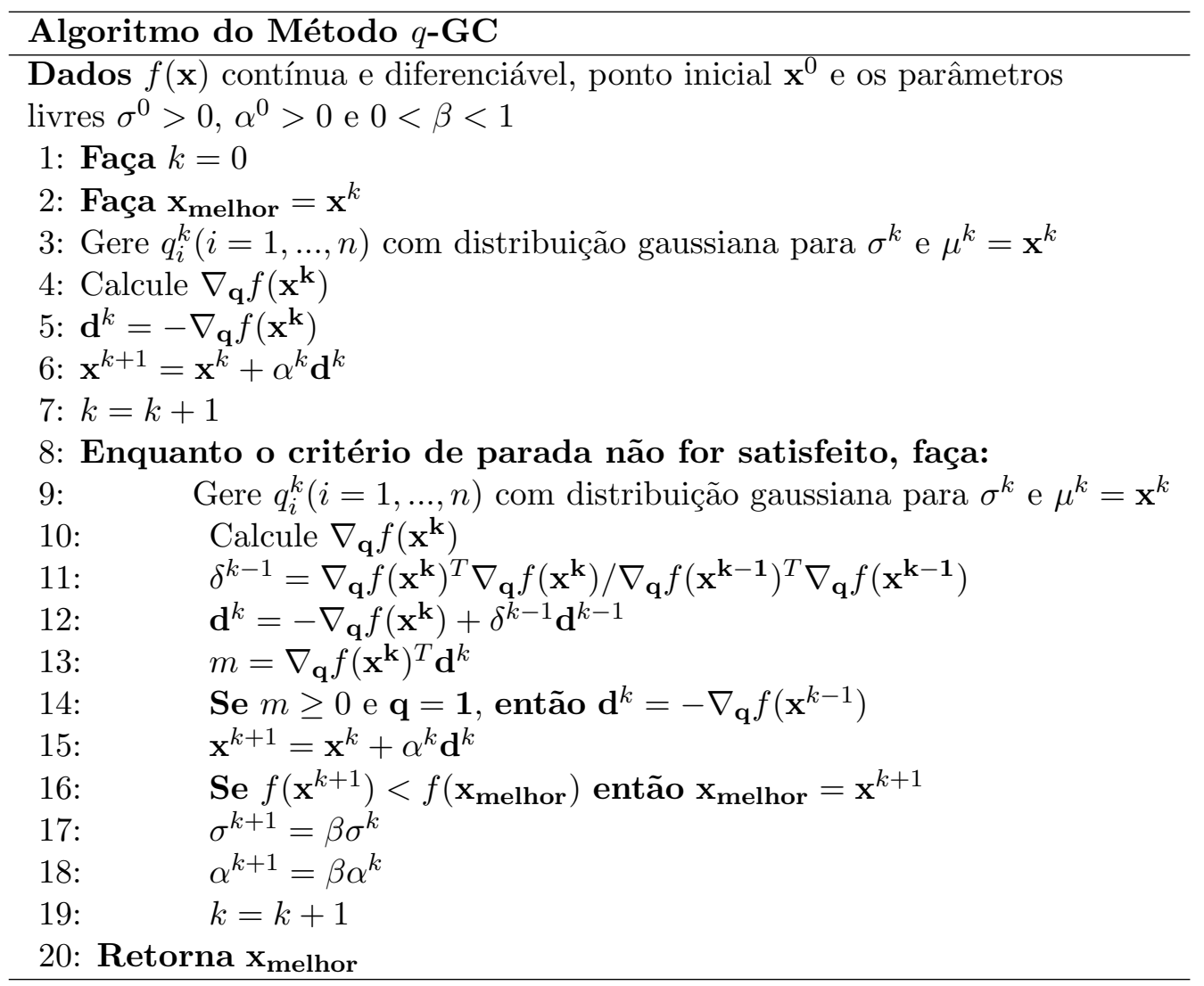

O algoritmo termina quando um critério de parada apropriado é satisfeito. Em aplicações do mundo real, onde o mínimo global não é conhecido, o critério de parada poderá ser um número máximo de avaliações da função objetivo ou o valor do gradiente da função no ponto atual $\left\|\nabla f\left(\mathbf{x}^{k}\right)\right\|$, desde que o método $q$-GC retome sua versão clássica no final da busca. O algoritmo retorna o melhor ponto visitado durante o procedimento iterativo como mostra o passo 20 do algoritmo. Note que as direções são reinicializadas apenas se a direção for de subida e o parâmetro q for igual a $\mathbf{1}$ (passo 14). O método q-GC utiliza apenas três parâmetros de ajuste: $\sigma^{0}, \alpha^{0}$ e $\beta$. Embora uma má escolha desses parâmetros causem uma deteriorização em seu desempenho, simulações mostraram que o algoritmo do método $q$-GC é suficientemente robusto sendo capaz de atingir a bacia de atração do mínimo global ${ }^{1}$. A seguir, o método $q$-GC é comparado com o método $q-\mathrm{G}$ e com AGs considerados eficientes na resolução de problemas de otimização global.

\section{Experimentos Computacionais}

O desempenho do método q-GC é avaliado em seis funções teste de 20 variáveis. As funções unimodais são: Ellipsoidal, Schwefel e Rosenbrock; e as funções multimodais são: Ackley, Rastrigin e Rastrigin Rotacionada. A descrição completa dessas funções pode ser encontrada em [10]. Todas as funções possuem mínimo global em $f\left(\mathbf{x}^{*}\right)=0$ com $\mathbf{x}^{*}=\mathbf{0}$, com exceção da função Rosembrock onde $\mathbf{x}^{*}=1$. Os critérios de parada são: máximo de $10^{6}$ avaliações da função objetivo ou $f(\mathbf{x})<10^{-20}$, os mesmos adotados em [1], [2] e [10]. A Tabela 1 exibe os valores dos parâmetros de ajuste $\sigma^{0}, \alpha^{0}$ e $\beta$, usados em cada função pelo método $q$-GC. Os valores de $\sigma^{0}$ e $\alpha^{0}$ foram normalizados pelo maior comprimento linear do espaço de busca, $L=\sqrt{\sum_{i=1}^{n}\left(\mathbf{x}_{\max _{i}}-\mathbf{x}_{\min _{i}}\right)^{2}}$, com $\mathbf{x}_{\max _{i}}, \mathbf{x}_{\min _{i}}$ definidos como em [1].

Os resultados são apresentados nas Tabelas 2 e 3. As colunas "Melhor", "Mediano" e "Pior"

\footnotetext{
${ }^{1}$ Bacia de atração do mínimo global $\mathbf{x}^{*}, \mathbf{x}^{*} \in \mathbb{R}^{n}$, de uma função $f(\mathbf{x})$ é a região $B, B \subset \mathbb{R}^{n}$, que contém o ponto $\mathbf{x}^{*}$ e, para todo $\mathbf{x} \in B$, uma trajetória de descida da $f(\mathbf{x})$ converge para o ponto $\mathbf{x}^{*}$ (veja [5]).
} 


\begin{tabular}{cccc}
\hline Funções & $\sigma^{\mathbf{0}} / L$ & $\alpha^{\mathbf{0}} / L$ & $\beta$ \\
\hline Ellipsoidal & 0,0012 & 0,0750 & 0,9000 \\
Schwefel & 0,0025 & 0,0025 & 0,9900 \\
Rosenbrock & 0,0001 & 0,3052 & 0,9995 \\
\hline Ackley & 0,0092 & 0,0083 & 0,9000 \\
Rastrigin & 0,1953 & 0,0004 & 0,9995 \\
Rastrigin Rotacionada & 0,1074 & 0,0005 & 0,9995 \\
\hline
\end{tabular}

Tabela 1: Parâmetros usados pelo método q-GC para o conjunto de funções teste.

referem-se, respectivamente, ao menor, ao mediano e ao maior número de avaliações da função objetivo necessárias para atingir a precisão desejada de $10^{-20}$. A coluna " $f\left(\mathbf{x}_{\text {melhor }}\right)$ " exibe o melhor valor da função objetivo encontrado durante o procedimento iterativo. Note que, quando a precisão desejada não é atingida, o melhor valor encontrado pelo método é exibido. A coluna "Sucesso" exibe o número de execuções independentes que atingiram a precisão desejada (para as funções unimodais) ou que alcançaram a bacia de atração do mínimo global (para as funções multimodais). Os melhores valores estão em negrito.

\begin{tabular}{llccccc}
\hline Função & Algoritmos & Melhor & Mediano & Pior & $f\left(\mathbf{x}_{\text {melhor }}\right)$ & Sucesso \\
\hline \multirow{5}{*}{ Ellipsoidal } & G3-PCX & $\mathbf{5 . 8 2 6}$ & $\mathbf{6 . 8 0 0}$ & $\mathbf{7 . 7 2 8}$ & $\mathbf{1 0}^{-\mathbf{2 0}}$ & $\mathbf{1 0 / 1 0}$ \\
& SPC-vSBX & 49.084 & 50.952 & 57.479 & $10^{-20}$ & $10 / 10$ \\
& SP-gradiente & $\mathbf{5 . 9 0 5}$ & $\mathbf{7 . 0 5 3}$ & $\mathbf{7 . 3 8 1}$ & $\mathbf{1 0}^{-\mathbf{2 0}}$ & $\mathbf{5 0 / 5 0}$ \\
& q-GC & 8.914 & 9.260 & 9.486 & $10^{-20}$ & $50 / 50$ \\
\hline \multirow{5}{*}{ Schwefel } & G3-PCX & $\mathbf{1 3 . 9 8 8}$ & $\mathbf{1 5 . 6 0 2}$ & $\mathbf{1 7 . 1 8 8}$ & $\mathbf{1 0}^{-\mathbf{2 0}}$ & $\mathbf{1 0 / 1 0}$ \\
& SPC-vSBX & 260.442 & 294.231 & 334.743 & $10^{-20}$ & $10 / 10$ \\
& SPC-PNX & 236.342 & 283.321 & 299.301 & $10^{-20}$ & $10 / 10$ \\
& q-gradiente & 289.174 & 296.103 & 299.178 & $10^{-20}$ & $50 / 50$ \\
& $q$-GC & 81.913 & 83.117 & 84.311 & $10^{-20}$ & $50 / 50$ \\
\hline \multirow{5}{*}{ Rosenbrock } & GPC-PNX & $10^{6}$ & - & - & $10^{-10}$ & $38 / 50$ \\
& SPC-vSBX & $\mathbf{1 6 . 5 0 8}$ & $\mathbf{2 1 . 4 5 2}$ & $\mathbf{2 5 . 5 2 0}$ & $\mathbf{1 0}^{-\mathbf{2 0}}$ & $\mathbf{3 6 / 5 0}$ \\
& q-gradiente & $10^{6}$ & - & - & $10^{-10}$ & $50 / 50$ \\
& $q$-GC & $10^{6}$ & - & - & $10^{-10}$ & $50 / 50$ \\
\hline
\end{tabular}

Tabela 2: Comparação entre os algoritmos sobre o conjunto de funções teste unimodais.

Para as funções unimodais (Tabela 2), o método $q$-GC apresenta um desempenho superior ao método $q$-G e dos AGs SPC-vSBX e SPC-PNX, mas é superado pelo AG G3-PCX. Note que o método $q$-GC atinge a precisão desejada em todas as 50 execuções. Para as funções multimodais (Tabela 3), tanto o método $q$-GC quanto o método $q-\mathrm{G}$ são claramente superiores aos AGs. Porém, o método $q$-GC é melhor que o método $q$-G pois atinge a precisão desejada com um maior número de sucessos ou um menor número de avaliações da função objetivo. Para a função Rastrigin Rotacionada, por exemplo, enquanto os três AGs foram incapazes de encontrar a bacia do mínimo global, o método $q$-GC conseguiu atingir essa bacia em $84 \%$ das execuções, contra 
Proceeding Series of the Brazilian Society of Applied and Computational Mathematics, Vol. 3, N. 1, 2015.

\begin{tabular}{llccccc}
\hline Função & Algoritmos & Melhor & Mediano & Pior & $f\left(\mathbf{x}_{\text {melhor }}\right)$ & Sucesso \\
\hline \multirow{5}{*}{ Ackley } & G3-PCX & $10^{6}$ & - & - & 3.959 & 0 \\
& SPC-vSBX & 57.463 & 63.899 & 65.902 & $10^{-10}$ & $10 / 10$ \\
& SPC-PNX & 45.736 & 48.095 & 49.392 & $10^{-10}$ & $10 / 10$ \\
& $q$-gradiente & 11.850 & 12.465 & 13.039 & $10^{-15}$ & $50 / 50$ \\
& $q$-GC & $\mathbf{1 1 . 2 9 5}$ & $\mathbf{1 1 . 4 2 4}$ & $\mathbf{1 1 . 5 2 2}$ & $\mathbf{1 0}^{-\mathbf{1 5}}$ & $\mathbf{5 0 / 5 0}$ \\
\hline \multirow{5}{*}{ Rastrigin } & G3-PCX & $10^{6}$ & - & - & 15.936 & 0 \\
& SPC-vSBX & 260.685 & 306.819 & 418.482 & $10^{-20}$ & $6 / 10$ \\
& SPC-PNX & $10^{6}$ & - & - & 4.975 & 0 \\
& $q$-gradiente & 676.050 & 692.450 & 705.037 & $10^{-20}$ & $48 / 50$ \\
& $q$-GC & $\mathbf{7 7 8 . 8 2 4}$ & $\mathbf{7 9 6 . 6 6 2}$ & $\mathbf{8 1 4 . 6 9 8}$ & $\mathbf{1 0}^{-\mathbf{2 0}}$ & $\mathbf{5 0 / 5 0}$ \\
\hline \multirow{5}{*}{ Rastrigin } & G3-PCX & $10^{6}$ & - & - & 309.429 & 0 \\
& SPC-vSBX & $10^{6}$ & - & - & 8.955 & 0 \\
& SPC-PNX & $10^{6}$ & - & - & 3.980 & 0 \\
\hline & $q$-gradiente & 541.857 & 545.957 & 549.114 & $10^{-20}$ & $20 / 50$ \\
& $q$-GC & $\mathbf{8 0 3 . 4 8 5}$ & $\mathbf{8 2 4 . 4 8 2}$ & $\mathbf{8 5 7 . 9 0 9}$ & $\mathbf{1 0}^{-\mathbf{2 0}}$ & $\mathbf{4 2 / 5 0}$ \\
\hline
\end{tabular}

Tabela 3: Comparação entre os algoritmos sobre o conjunto de funções teste multimodais.

apenas $40 \%$ apresentado pelo $q-\mathrm{G}$.

\section{Conclusões}

Este trabalho apresenta uma $q$-versão do método dos gradientes conjugados, denominada método $q$-GC, baseado no conceito do vetor $q$-gradiente desenvolvido em [10]. A principal ideia por trás do método é a transição entre busca global, no início, e busca local, no final, do procedimento iterativo, com a presença de mecanismos que permitem que o método escape de mínimos locais e caminhe cada vez mais na direção do mínimo global. O método $q$-GC foi comparado com o método $q-\mathrm{G}$ (veja [10]) e com três AGs (veja $[1,2]$ ), para seis funções teste da literatura. Embora preliminares, o desempenho do método $q$-GC é promissor, especialmente quando aplicado em funções multimodais.

\section{Referências}

[1] P. J. Ballester e J. N. Carter, An effective real-parameter genetic algorithm with parent centric normal crossover for multimodal optimisation, em "Genetic and Evolutionary Computation Conference - GECCO 2004" (K. Deb, ed.) pp. 901-913, Springer-Verlag, Seatle, WA, 2004.

[2] K. Deb, A. Anand e D. Joshi, A computationally efficient evolutionary algorithm for realparameter optimization, Evolutionary Computation, 10 (2002) 345-369.

[3] G. Di Pillo e L. Palagi, Nonlinear programming: Introduction, em "Handbook of applied optimization" (P. M. Pardalos e M. G. C. Resende) pp. 263-268, Oxford University Press, New York, 2002. 
[4] R. Fletcher e C. M. Reeves, Function minimization by conjugate gradients, Computer Journal, 07 (1964) 149-154.

[5] H. Huang, Global Optimization: Filled Function Methods, em "Encyclopedia of Optimization, Second Edition"(C. A. Floudas e P. M. Pardalos, eds.) pp. 1316-1323, Springer, New York, 2009.

[6] F. H. Jackson, On $q$-functions and a certain difference operator, Trans. Roy Soc. Edin., 46 (1908) 253-281.

[7] F. H. Jackson, On q-definite integrals, Quart. J. Pure and Appl. Math., 41 (1910) 193-203.

[8] F. H. Jackson, q-Difference Equations, American Journal of Mathematics, 32, (1910) 307314.

[9] J. Nocedal e S. J. Wright, "Numerical Optimization", Springer-Verlag, New York, 1999.

[10] A. C. Soterroni, R. L. Galski e F. M. Ramos, The q-gradient method for global optimization, arXiv:1209.2084, math.OC (2012).

[11] G. N. Vanderplaats, "Numerical optimization techniques for engineering design", McGrawHill, New York, 1984. 